\title{
Improvement in EMI Shielding Properties of Silicone Rubber/POE Blends Containing ILs Modified with Carbon Black and MWCNTs
}

\author{
Chao Liu, Chuyang Yu, Guolong Sang, Pei Xu * and Yunsheng Ding * \\ School of Chemistry and Chemical Engineering, and Anhui Key Laboratory of Advanced Functional Materials \\ and Devices, Hefei University of Technology, Hefei 230009, China; f62889@163.com (C.L.); \\ Ycy19990913@163.com (C.Y.); sglforget@163.com (G.S.) \\ * Correspondence: chxuper@hfut.edu.cn (P.X.); dingys@hfut.edu.cn (Y.D.); Tel.: +86-551-62901545 (Y.D.)
}

Received: 27 March 2019; Accepted: 22 April 2019; Published: 29 April 2019

check for updates

Featured Application: The present study provides an ingenious and effective method to fabricate a promising and flexible composites for electromagnetic interference shielding applications.

\begin{abstract}
Silicone rubber (SR)/polyolefin elastomer (POE) blends containing ionic liquids modified with carbon blacks (CB-IL) and multi-walled carbon nanotubes (CNT-IL) were prepared by melt-blending and hot pressing. SR/POE/CB-IL and SR/POE/CB-CNT-IL composites showed co-continuous structural morphologies. The cation $-\pi$ interactions between ILs and CNTs were stronger than those between ILs and CBs due to the large length and high surface area of CNTs, which promoted better dispersion of carbon fillers. SR/POE/CB-CNT-IL composites showed higher EMI $\mathrm{SE}$ than SR/POE/CB-IL composites containing identical filler contents because the CNTs with larger aspect ratios helped form more electrically-conductive networks.
\end{abstract}

Keywords: polymeric composites; silicone rubber; Ionic liquid; carbon materials; structural; EMI shielding

\section{Introduction}

In the rapidly-growing information age, silicone rubber has seen extensive application in the electronic and electrical equipment industries due to its excellent chemical resistance, non-toxic nature, heat-resistance, freeze-resistance, and good flexibility [1]. However, silicone rubber is electrically-insulating and transparent to electromagnetic radiation. As electronic information technology has developed and the use of electronic devices has become more widespread, electromagnetic interference (EMI), which interrupts the functionality of electronic devices and severely affects human organs, has become a serious problem [2-4]. Therefore, conductive rubber composites containing carbon materials, such as carbon blacks and multi-walled carbon nanotubes, have excellent electrical conductivities and flexibility and have been widely used as EMI shielding materials $[5,6]$. A typical approach is to add a single type of carbon material into a single kind of polymeric matrix to increase the shielding ability of the carbon-based polymer composites [7-10]. Ionic liquids (ILs), which consist of a pair of soft cationic and anionic species, are used to improve the dispersion of carbon fillers in the polymer matrix since ILs and carbon fillers exhibit strong cation $-\pi$ physical interactions [11-14].

In this study, carbon blacks (CBs) and carbon nanotubes (CNTs) were modified with 1-vinyl-3-ethylimidazolium bromide ILs that contained carbon double bonds. The synergistic effect of ILs modified with CBs and CNTs on the morphology and the EMI shielding properties of silicone rubber/polyolefin elastomer/carbon black-carbon nanotube-ionic liquid (SR/POE/CB-CNT-IL) 
composites were systematically investigated to reveal the mechanism of the enhanced EMI shielding performance.

\section{Experimental}

Methyl vinyl silicone rubber (SR) with a vinyl content of $0.16 \mathrm{wt} \%$ was purchased from Wynca Co., Ltd., China. POE (grade 8150), with a melt flow index of $0.5 \mathrm{~g} / 10 \mathrm{~min}$ at $190^{\circ} \mathrm{C} / 2.16 \mathrm{~kg}$, was purchased from Dow Chemical Co., Ltd., USA. Conductive carbon blacks (CBs) with a BET surface area of $83 \mathrm{~m}^{2} / \mathrm{g}$ were purchased from Evonik Degussa Co., Ltd., Germany. Multi-walled carbon nanotubes (CNTs, $10-20 \mathrm{~nm}$ in internal diameter and 10-30 $\mu \mathrm{m}$ in length) were supplied by Nanjing Xianfeng Nano Material Technology Co., Ltd., China. The 1-vinyl-3-ethylimidazolium bromide ILs were supplied by Lanzhou Greenchem ILs Co., Ltd., China. 2,5-dimethyl-2,5-di(tert-butylper-oxy)-hexane (DBPH) as curing agent was purchased from AkzoNobel Peroxide Co., China. EVA (ethylene-vinylacetate copolymer, grade $265,28 \mathrm{wt} \%$ VA content) was purchased from Dupont Co., USA, and was used as a non-reactive compatibilizer to enhance the interfacial adhesion between SR and POE.

The SR/POE/CB-CNT-IL composites were prepared as follows. First, CBs, CBs, and CNTs, ILs-modified CBs (CB-IL), ILs-modified CBs and CNTs by (CB-CNT-IL) were ground in an agate mortar. Then, the fillers were added to SR by a two-roll mill at $125^{\circ} \mathrm{C}$, and POE and DBPH were introduced and mixed for $15 \mathrm{~min}$. Finally, the composites were vulcanized under $10 \mathrm{MPa}$ pressure for $10 \mathrm{~min}$ at $180^{\circ} \mathrm{C}$, and then post-cured in a drying oven at $190^{\circ} \mathrm{C}$ for $3 \mathrm{~h}$. The amounts of components prepared for the composites are shown in Table 1.

Table 1. Composite compositions (weight ratio).

\begin{tabular}{cccccccc}
\hline Sample & SR & POE & CB & MWCNTs & ILs & EVA & DBPH \\
\hline SR/POE & 60 & 40 & - & - & - & 5 & 2 \\
SR/POE/5CB-IL & 60 & 40 & 5 & - & 1 & 5 & 2 \\
SR/POE/10CB-IL & 60 & 40 & 10 & - & 2 & 5 & 2 \\
SR/POE/15CB-IL & 60 & 40 & 15 & - & 3 & 5 & 2 \\
SR/POE/20CB & 60 & 40 & 20 & - & - & 5 & 2 \\
SR/POE/20CB-IL & 60 & 40 & 20 & - & 4 & 5 & 2 \\
SR/POE/15CB-CNT-IL & 60 & 40 & 10 & 5 & 3 & 5 & 2 \\
SR/POE/20CB-CNT & 60 & 40 & 15 & 5 & - & 5 & 2 \\
SR/POE/20CB-CNT-IL & 60 & 40 & 15 & 5 & 4 & 5 & 2 \\
\hline
\end{tabular}

The morphology of the composites was observed by scanning electron microscopy (SEM, JEOL, JSM-6490LV, Japan) at an accelerating voltage of $20 \mathrm{kV}$ and field-emission scanning electron microscopy (FESEM, JSM-6700F) at an accelerating voltage of $5 \mathrm{kV}$. The electrical conductivity was determined using a multi-function digital electric meter (Victor Tech, Victor 86-e). The EMI shielding effectiveness (SE) data were obtained by using nanocomposite slabs $\left(22.86 \times 10.16 \times 0.6 \mathrm{~mm}^{3}\right)$, which were fitted into a waveguide sample holder with a vector network analyser (Agilent Technologies, N5247A, America) at 8.0 to $12.0 \mathrm{GHz}$ ( $\mathrm{X}$ band). EMI shielding properties of the samples were evaluated from the $\mathrm{S}$ parameters, which could be calculated calculate $\mathrm{SE}_{\mathrm{T}}$ (total shielding effectiveness), $\mathrm{SE}_{\mathrm{R}}$ (reflection loss) and $\mathrm{SE}_{\mathrm{A}}$ (absorption loss) [15].

\section{Results and Discussion}

Figure 1 shows the SEM images of the fractured surfaces of SR/POE/CB-IL and SR/POE/CB-CNT-IL composites. The samples were first fractured in liquid nitrogen, and then the fractured surfaces were immersed into n-hexane at $90{ }^{\circ} \mathrm{C}$ for $12 \mathrm{~h}$ to remove the POE phase. All samples display typical co-continuous structures, and the collapsed surface morphology is attributed to the dissolution of the POE phase. Carbon fillers modified by ILs were premixed with SR and then mixed with POE. Most fillers were initially dispersed in the SR matrix because the localization of fillers is controlled by flow properties and dynamic factors. When CNTs were added, the viscosity of the SR phase increased 
significantly, and the volume fraction of the SR phase decreased significantly due to the large length and high surface area of CNTs. This caused the SR/POE/CB-CNT-IL composites to form regular and orderly co-continuous phase structures [16].
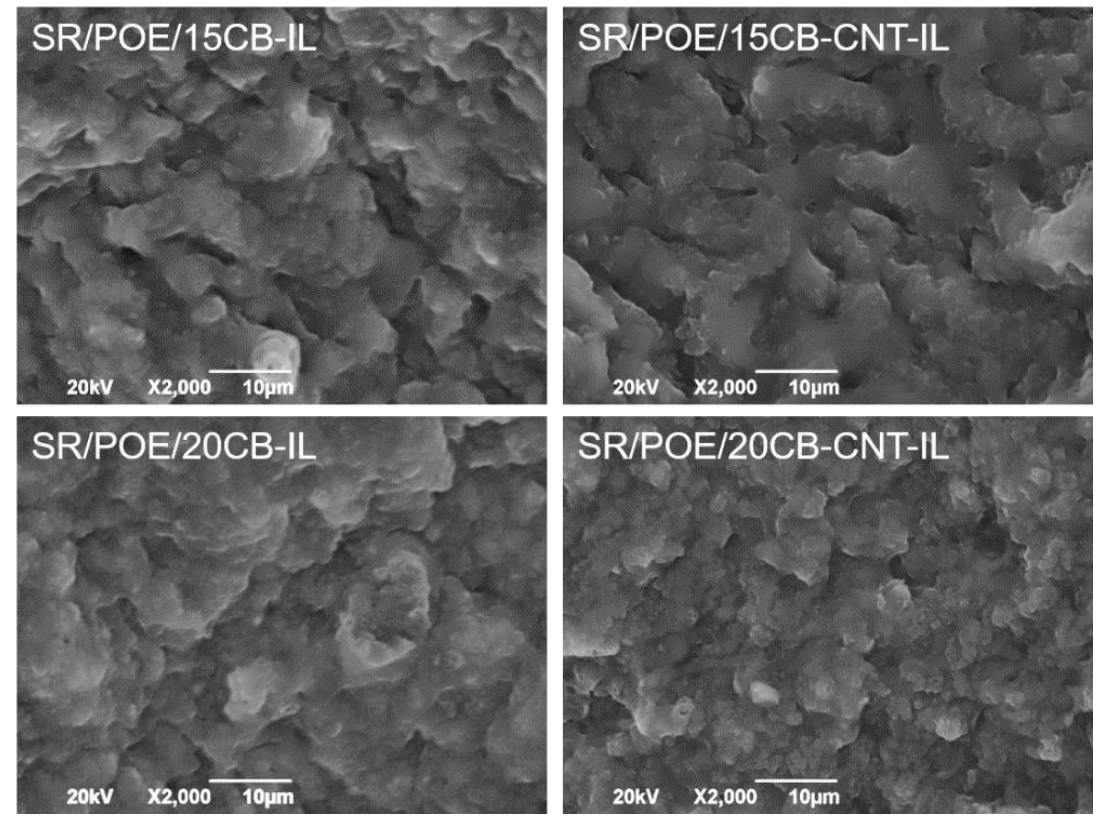

Figure 1. Scanning electron microscopy (SEM) images of the fracture surfaces of the silicone rubber/polyolefin elastomer/carbon black -ionic liquid (SR/POE/CB-IL) and silicone rubber/polyolefin elastomer/carbon black-carbon nanotube-ionic liquid (SR/POE/CB-CNT-IL) composites.

Figure 2 shows the FESEM images of the fracture surfaces of the SR/POE/CB-IL, SR/POE/CB, SR/POE/CB-CNT-IL and SR/POE/CB-CNT composites. When the ILs were added, the dispersion of carbon filler was improved significantly because the cation $-\pi$ interactions between ILs and carbon fillers promoted the further dispersion of fillers and reduced agglomeration. In addition, when the CNTs were added, the cation- $\pi$ interactions between ILs and CNTs were stronger than those between ILs and CBs due to the large length and high surface area of CNTs [17].
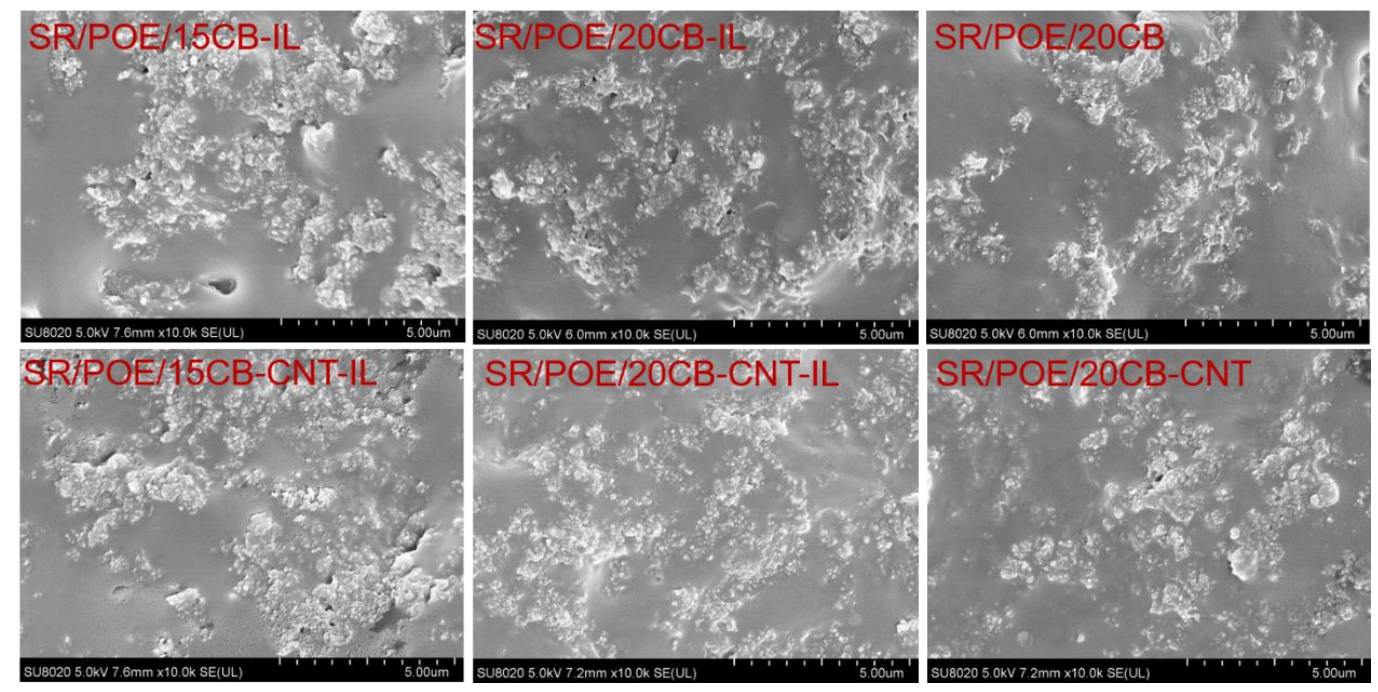

Figure 2. Field-emission scanning electron microscopy (FESEM) images of the fracture surfaces of the SR/POE/CB-IL, SR/POE/CB, SR/POE/CB-CNT-IL, and SR/POE/CB-CNT composites. 
As the CB-IL content increased, the electrical conductivity of the SR/POE/15CB-IL and SR/POE/20CB-IL composites was $3.6 \times 10^{-1}$ and $8.9 \times 10^{-1} \mathrm{~S} / \mathrm{m}$, respectively. For the SR/POE/CB-CNT-IL composites, as the CB-CNT-IL content increased, the conductivity of the SR/POE/15CB-CNT-IL and SR/POE/20CB-CNT-IL composites was $6.7 \times 10^{-1}$ and $3.5 \mathrm{~S} / \mathrm{m}$, respectively. CB-CNT-IL can regulate and control the aggregation, dispersion and uniformity of carbon fillers to form three-dimensional conductive networks. This improves the electrical conductivity, and the EMI SE values increased as the amount of fillers in the composites increased. Figure 3a shows the EMI shielding effectiveness (SE) of the composites in the frequency range of 8.0 to $12.0 \mathrm{GHz}$. SR/POE/CB-CNT-IL composites have higher EMI SE values than the SR/POE/CB-IL composites at identical filler loadings [18]. The CNTs with larger aspect ratios helped form a more conductive network and improved the EMI performance of the SR/POE/CB-CNT-IL composites. Specifically, the shielding effectiveness of the SR/POE/20CB-IL and SR/POE/20CB-CNT-IL composites was $29.4 \mathrm{~dB}$ and $36.5 \mathrm{~dB}$, over the frequency range of 8.0 to $12.0 \mathrm{GHz}$, respectively. This gives the SR/POE/20CB-CNT-IL composite a novel potential electromagnetic interference that shields materials by eliminating electromagnetic pollution over a wide frequency range. To correlate the EMI shielding properties with the composite structures, both the average $\mathrm{SE}_{\mathrm{T}}, \mathrm{SE}_{\mathrm{A}}$, and $\mathrm{SE}_{\mathrm{R}}$ values of SR/POE/CB-IL and SR/POE/CB-CNT-IL composites at $9.0 \mathrm{GHz}$ were plotted with filler content as shown in Figure $3 \mathrm{~b}$. The $\mathrm{SE}_{\mathrm{A}}$ and $\mathrm{SE}_{\mathrm{R}}$ showed an increase as the filler content increased due to the synergistic effect between ILs and carbon fillers, which enhanced the electrical conductivity. Furthermore, the contribution of absorption to $\mathrm{SE}_{\mathrm{T}}$ is greater than that of the reflection because network conductivity supports abundant interfaces which form multiple reflections and attenuate the incident EM waves [19]. The filler content in the SR/POE/CB-CNT-IL composites should be well above their percolation thresholds. Herein, the excellent EMI SE of the $\mathrm{SR} / \mathrm{POE} / \mathrm{CB}-\mathrm{CNT}-\mathrm{IL}$ composites is attributed to the presence of highly conductive networks, which effectively reflect, multi-scatter and adsorb the incident radiations. Figure 4a presents the $\mathrm{SE}_{\mathrm{T}}, \mathrm{SE}_{\mathrm{A}}$, and $\mathrm{SE}_{\mathrm{R}}$ of SR/POE/CB, SR/POE/CB-CNT, SR/POE/CB-IL and SR/POE/CB-CNT-IL composites at 9.0 GHz. The EMI shielding performance of $\mathrm{SR} / \mathrm{POE} / \mathrm{CB}$ and $\mathrm{SR} / \mathrm{POE} / \mathrm{CB}-\mathrm{CNT}$ composites obviously decreased compared with that of SR/POE/CB-IL and SR/POE/CB-CNT-IL composites. The results reveal that ILs promote the dispersion of carbon fillers due to the cation- $\pi$ interactions between ILs and carbon fillers. Compared to SR/POE/CB-IL composites, the SR/POE/CB-CNT-IL composites with co-continuous structures have enhanced carrier mobility due to the presence of denser conductive networks and more interfacial structures formed by the uniformly-dispersed CB-CNT-IL. The schematic illustration of EMI shielding mechanisms for the SR/POE/CB-CNT-IL composites is shown in Figure $4 \mathrm{~b}$. The excellent EMI SE in this work can be attributed to the highly assembled carbon nano-fillers conductive networks that effectively reflect, multiple scatter and adsorb the incident radiation [20].
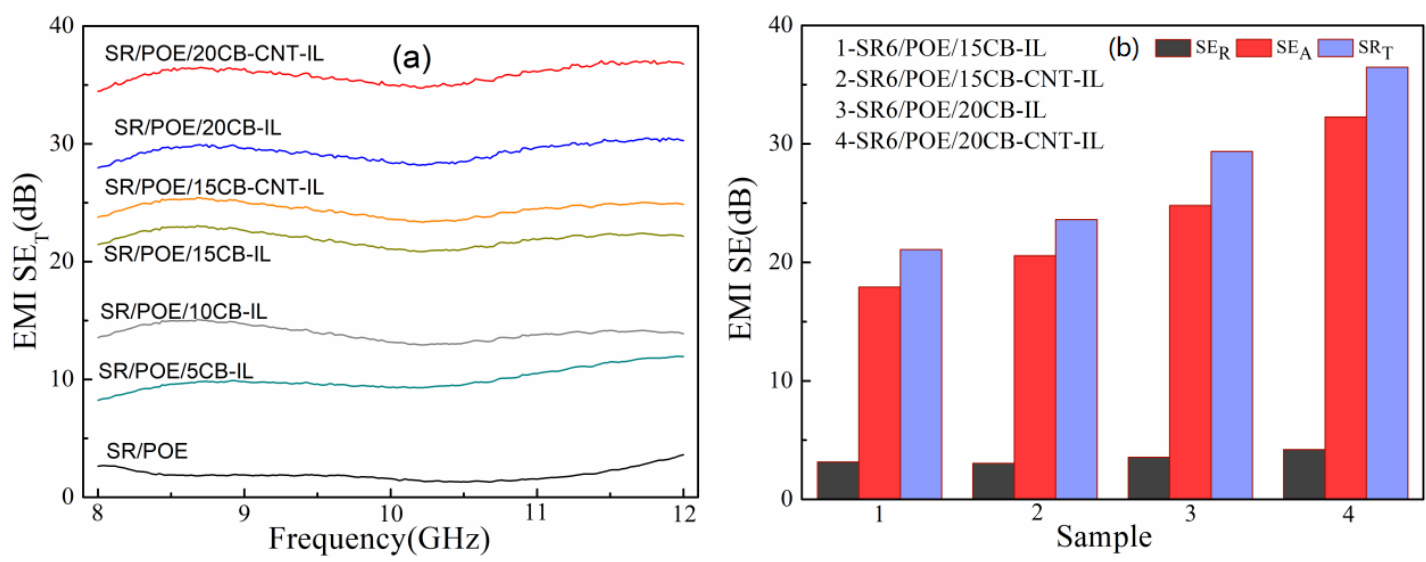

Figure 3. (a) Electromagnetic interference shielding effectiveness (EMI SE) of the SR/POE/CB-IL and $\mathrm{SR} / \mathrm{POE} / \mathrm{CB}-\mathrm{CNT}-\mathrm{IL}$ composites in the frequency range of 8.0 to $12.0 \mathrm{GHz}$, and (b) comparison of $\mathrm{SE}_{\mathrm{T}}$, $\mathrm{SE}_{\mathrm{A}}$, and $\mathrm{SE}_{\mathrm{R}}$ of the SR/POE/CB-IL and SR/POE/CB-CNT-IL composites at $9.0 \mathrm{GHz}$. 


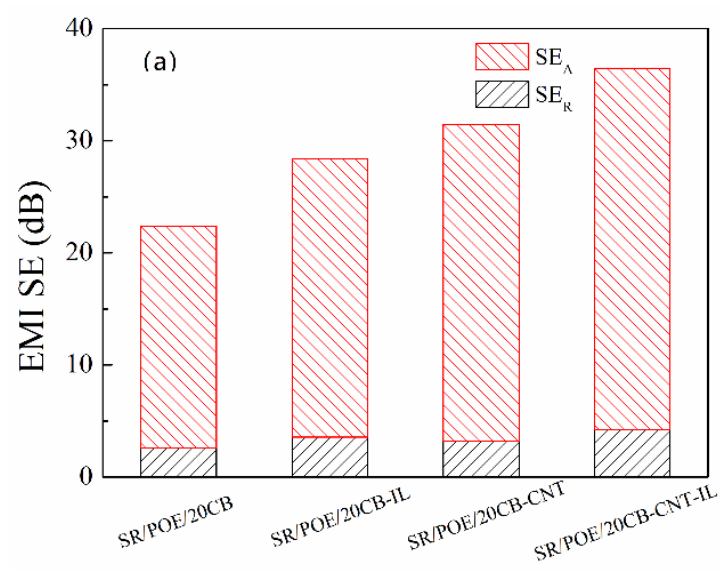

(b)
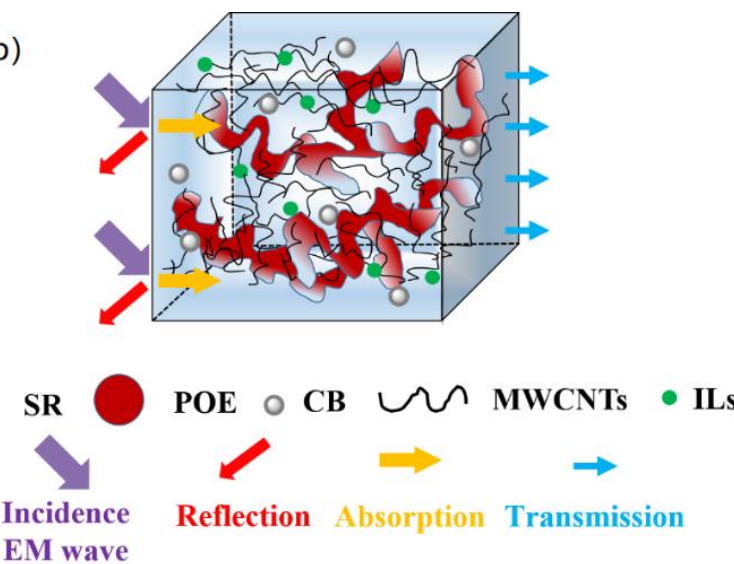

Figure 4. (a) Comparison of $\mathrm{SE}_{\mathrm{T}}, \mathrm{SE}_{\mathrm{A}}$, and $\mathrm{SE}_{\mathrm{R}}$ of the $\mathrm{SR} / \mathrm{POE} / \mathrm{CB}, \mathrm{SR} / \mathrm{POE} / \mathrm{CB}-\mathrm{IL}, \mathrm{SR} / \mathrm{POE} / \mathrm{CB}-\mathrm{CNT}-\mathrm{IL}$, and $\mathrm{SR} / \mathrm{POE} / \mathrm{CB}-\mathrm{CNT}$ composites at $9.0 \mathrm{GHz}$, and $(\mathbf{b})$ Schematic illustration of EMI shielding mechanisms for the SR/POE/CB-CNT-IL composites.

\section{Conclusions}

In summary, this study has investigated the morphology and EMI shielding properties of SR/POE/CB-CNT-IL composites, which were fabricated by a conventional two-roll mill mixing process. The cation $-\pi$ interactions between ILs and CNTs were stronger than those between ILs and CBs due to the large length and surface areas of CNTs which promoted further dispersion of carbon fillers. The conductive networks play an important role in reflecting, scattering multiplication and absorption of the incident radiation. The SR/POE/CB-CNT-IL composites exhibited excellent EMI shielding properties due to the synergistic effect of ILs-modified CBs and CNTs. The EMI SE of the SR/POE/20CB-CNT-IL composite could reach $36.5 \mathrm{~dB}$ at $9.0 \mathrm{GHz}$. This gives the SR/POE/20CB-CNT-IL composite a novel potential electromagnetic interference that shields materials by eliminating electromagnetic pollution over a wide frequency range.

Author Contributions: Validation, G.S.; investigation, C.Y.; writing-original draft preparation, C.L.; writing—review and editing, P.X.; supervision and project administration, Y.D.

Funding: This research was funded by the Anhui Provincial Science and Technology Major Project (17030901074).

Conflicts of Interest: The authors declare no conflict of interest.

\section{References}

1. Li, Y.L.; Li, M.J.; Pang, M.L.; Feng, S.Y.; Zhang, J.; Zhang, C.Q. Effects of multi-walled carbon nanotube structures on the electrical and mechanical properties of silicone rubber filled with multi-walled carbon nanotubes. J. Mater. Chem. C 2015, 3, 5573-5579. [CrossRef]

2. Wang, H.B.; Teng, K.Y.; Chen, C.; Li, X.J.; Xu, Z.W.; Chen, L.; Fu, H.J.; Kuang, L.Y.; Ma, M.J.; Zhao, L.H. Conductivity and electromagnetic interference shielding of graphene-based architectures using MWCNTs as free radical scavenger in gamma-irradiation. Mater. Lett. 2017, 186, 78-81. [CrossRef]

3. Wang, G.L.; Wang, L.; Mark, L.H.; Shaayegan, V.; Wang, G.Z.; Li, H.P.; Zhao, G.Q.; Park, C.B. Ultralow-threshold and lightweight biodegradable porous PLA/MWCNT with segregated conductive networks for high-performance thermal insulation and electromagnetic interference shielding applications. ACS Appl. Mater. Inter. 2018, 10, 1195-1203. [CrossRef] [PubMed]

4. Engels, S.; Schneider, N.L.; Lefeldt, N.; Hein, C.M.; Zapka, M.; Michalik, A.; Elbers, D.; Kittel, A.; Hore, P.J.; Mouritsen, $\mathrm{H}$. Anthropogenic electromagnetic noise disrupts magnetic compass orientation in a migratory bird. Nature 2014, 509, 353-356. [CrossRef] [PubMed]

5. Wang, R.; Yang, H.; Wang, J.L.; Li, F.X. The electromagnetic interference shielding of silicone rubber filled with nickel coated carbon fiber. Polym. Test. 2014, 38, 53-56. [CrossRef] 
6. Sudha, J.D.; Sivakala, S.; Kamlesh, P.; Nair, P.R. Development of electromagnetic shielding materials from the conductive blends of polystyrene polyaniline-clay nanocomposite. Compos. Part A Appl. Sci. Manuf. 2010, 41, 1647-1652. [CrossRef]

7. Das, N.C.; Khastgir, D.; Chaki, T.K.; Chakraborty, A. Electromagnetic interference shielding effectiveness of carbon black and carbon fibre filled EVA and NR based composites. Compos. Part A Appl. Sci. Manuf. 2000, 31, 1069-1081. [CrossRef]

8. Zeng, Z.H.; Jin, H.; Chen, M.J.; Li, W.W.; Zhou, L.C.; Zhang, Z. Lightweight and anisotropic porous MWCNT/WPU composites for ultrahigh performance electromagnetic interference shielding. Adv. Funct. Mater. 2016, 26, 303-310. [CrossRef]

9. Wang, G.L.; Zhao, G.Q.; Wang, S.; Zhang, L.; Park, C.B. Injection-molded microcellular PLA/graphite nanocomposites with dramatically enhanced mechanical and electrical properties for ultra-efficient EMI shielding applications. J. Mater. Chem. C 2018, 6, 6847-6859. [CrossRef]

10. Jin, L.; Zhao, X.M.; Xu, J.F.; Luo, Y.Y.; Chen, D.Q.; Chen, G.H. The synergistic effect of a graphene nanoplate/ $\mathrm{Fe}_{3} \mathrm{O}_{4} @ \mathrm{BaTiO}_{3}$ hybrid and MWCNTs on enhancing broadband electromagnetic interference shielding performance. RSC Adv. 2018, 8, 2065. [CrossRef]

11. Poothanari, M.A.; Abraham, J.; Kalarikkal, N.; Thomas, S. Excellent electromagnetic interference shielding and high electrical conductivity of compatibilized polycarbonate/polypropylene carbon nanotube blend nanocomposites. Ind. Eng. Chem. Res. 2018, 57, 4287-4297. [CrossRef]

12. Kowsari, E.; Mohammadi, M. Synthesis of reduced and functional graphene oxide with magnetic ionic liquid and its application as an electromagnetic-absorbing coating. Compos. Sci. Technol. 2016, 126, 106-114. [CrossRef]

13. Abraham, J.; Arif, M.P.; Kailas, L.; Kalarikkal, N.; George, S.C.; Thomas, S. Developing highly conducting and mechanically durable styrene butadiene rubber composites with tailored microstructural properties by a green approach using ionic liquid modified MWCNTs. RSC Adv. 2016, 6, 32493. [CrossRef]

14. Abraham, J.; Arif, M.P.; Xavier, P.; Bose, S.; George, S.C.; Kalarikkal, N.; Thomas, S. Investigation into dielectric behaviour and electromagnetic interference shielding effectiveness of conducting styrene butadiene rubber composites containing ionic liquid modified MWCNT. Polymer 2017, 112, 102-115. [CrossRef]

15. Li, Q.L.; Chen, L.; Ding, J.J.; Zhang, J.J.; Li, X.H.; Zheng, K.; Zhang, X.; Tian, X.Y. Open-cell phenolic carbon foam and electromagnetic interference shielding properties. Carbon 2016, 104, 90-105. [CrossRef]

16. Cao, M.; Shu, J.J.; Chen, P.; Xia, R.; Yang, B.; Miao, J.B.; Qian, J.S. Orientation of boron nitride nanosheets in CM/EPDM co-continuous blends and their thermal conductive properties. Polym. Test. 2018, 69, $208-213$. [CrossRef]

17. Wang, P.; Xu, P.; Zhou, Y.Y.; Yang, Y.W.; Ding, Y.S. Effect of MWCNTs and P[MMA-IL] on the crystallization and dielectric behavior of PVDF composites. Eur. Polym. J. 2018, 99, 58-64. [CrossRef]

18. Al-Saleh, M.H. Influence of conductive network structure on the EMI shielding and electrical percolation of carbon nanotube/polymer nanocomposites. Synth. Met. 2015, 205, 78-84. [CrossRef]

19. Sang, G.L.; Dong, J.W.; He, X.T.; Jiang, J.C.; Li, J.B.; Xu, P.; Ding, Y.S. Electromagnetic interference shielding performance of polyurethane composites: A comparative study of $\mathrm{GNs}-\mathrm{IL} / \mathrm{Fe}_{3} \mathrm{O}_{4}$ and $\mathrm{MWCNTs}-\mathrm{IL} / \mathrm{Fe}_{3} \mathrm{O}_{4}$ hybrid fillers. Compos. Part B Eng. 2019, 164, 467-475. [CrossRef]

20. Huang, H.D.; Liu, C.Y.; Zhou, D.; Jiang, X.; Zhong, G.J.; Yan, D.X.; Li, Z.M. Cellulose composite aerogel for highly efficient electromagnetic interference shielding. J. Mater. Chem. A 2015, 3, 4983-4991. [CrossRef]

(C) 2019 by the authors. Licensee MDPI, Basel, Switzerland. This article is an open access article distributed under the terms and conditions of the Creative Commons Attribution (CC BY) license (http://creativecommons.org/licenses/by/4.0/). 